\title{
EFFICIENT NEAR-INFRARED ELECTRO-IONIZATION LASERS
}

\author{
W. J. Witteman and P. J. M. Peters \\ University of T rente, Department of Applied Physics, P.O. Box 217, 7500 AE Enschede, The Netherlands
}

\begin{abstract}
High power near-infrared radiation $(1-3 \mu \mathrm{m})$ can be generated with electro-ionization lasers using rare gases at multi-atmosphere pressure. The best performance is obtained in a so-called e-beam sustained discharge where the e-beam is used to ionize the medium and to maintain the stability of the discharge. An intrinsic efficiency of $8 \%$ is feasible. The output power saturates with current due to electron collision mixing. A maximum of $8 \mathrm{~kW} / \mathrm{cm}^{3}$ has been observed.
\end{abstract}

\section{INTRODUCTION}

Laser action in low pressure glow discharges like the $\mathrm{He}-\mathrm{Ne}$ laser based on electronic transitions is characterized by low output power and low efficiency of the order of $10^{-3}$. The reason for this bad performance at low gas pressure is the prohibition of volume recombination of ions and electrons. The recombination takes only place at the discharge wall so that the discharge adjusts itself to an ion production that just balances these losses. This means that the average energy of the electrons in the discharge is low and that in inelastic collisions mainly electronic transitions are produced. The low power saturation involves the role of the metastable levels to which the terminal laser levels decay. These levels have long life times and will be destroyed by excitation to higher electronic states and by collisions with the wall. Increasing the current does not change the average electron energy but it results to the overcrowding at the metastable levels. This eventually creates an overcrowding of the lower laser levels because of the self absorption of the decaying lower laser level so that the depopulation rate of the lower laser levels decrease and consequently the output power.

At multi-atmosphere pressure the situation is very different. Molecular ions like $\mathrm{Ar}_{2}^{+}$and $\mathrm{ArXe}^{+}$ formed by three-body-collisions in a $\mathrm{Ar}-\mathrm{Xe}$ mixture play an essential role. Volume recombination as a three-body-process is then the main channel in the kinetic chain of the inversion mechanism. To balance the volume recombination the average electron energy in the discharge adjusts to a sufficient ion production mainly from the metastable states. An essential role in the kinetics is played by a buffer gas. The system works as a four level system (see Fig. 1) that uses the metastable state as the ground state in the excitation scheme. Because the ionization of these metastable states in the discharge is also the excitation step of the inversion process this type of laser is called an electro-ionization laser. After the ions recombine with the electrons they decay to form the upper laser level. The lower laser level on its turn decays to the metastable level. In this way it closes the cycle. Powerful high pressure rare gas lasers operating on electronic transitions can be obtained.

The technical problem to realize such a system is to find conditions for obtaining a stable discharge of high current at multi-atmospheric densities. Newman et al..$^{(1,2)}$ were the first who reported on the successful operation of this type of laser with an electrical efficiency of nearly $1 \%$. They used an e-beam sustained discharge for efficient pumping of a high pressure Ar-Xe mixture on the well-known infrared transitions $(1.73-3.51 \mu \mathrm{m})$ between the $5 \mathrm{~d}$ and $6 \mathrm{p}$ manifolds of xenon as shown in Fig. 2. The e-beam is primarily used to ionize the medium and to condition the stability of the discharge. The sustainer discharge ionizes the xenon atoms from the metastable state. After this success there has been considerable effort to realize efficient high power infrared laser systems on rare-gas atomic transitions. During a quasi continuous regime of about $3.5 \mu \mathrm{s}$ for the excitation of the infrared xenon transitions with an e-beam sustained system an intrinsic efficiency of $5 \%$ and an output of $8 \mathrm{~J} / 1$ is obtained..$^{(3)}$ Recently ${ }^{(4)}$ it was observed that e-beam sustained systems have the potential of even $8 \%$ intrinsic efficiency. This laser may become an attractive infrared source because only rare gases are involved so that in principle long lifetimes are expected. Molecular 


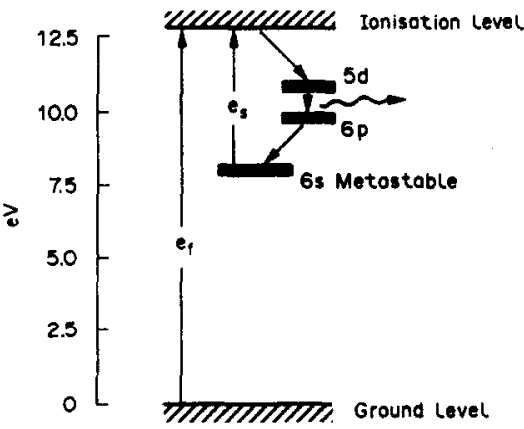

Fig. 1. Four level laser scheme of $\mathrm{Ar}-\mathrm{Xe}$.

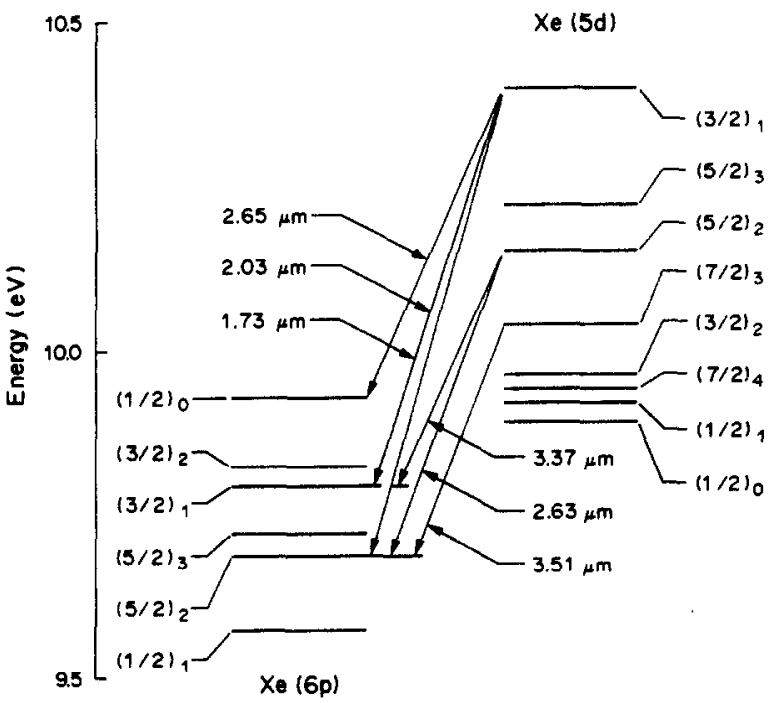

Fig. 2. Energy levels of the $5 \mathrm{~d}-6 \mathrm{p}$ manifolds of $\mathrm{Xe}$ showing the laser transitions. In $\mathrm{Ar}-\mathrm{Ze}$ mixtures the $1.73 \mu \mathrm{m}$ transition is the strongest.

infrared systems are mostly hampered by dissociation problems caused by the discharge. Another advantage of the atomic system is the absence of the thermal population of the lower laser level so that thermal saturation as in the case of $\mathrm{CO}_{2}$ lasers is no problem. Cooling provisions are less critical. On the other hand by trying to develop this atomic system to a practical device with a high average output the main problem is to find discharge conditions for these high pressure systems that allow for efficient inversion production at a high repetition rate. It has been shown that successful operation can occur in various modes such as electric discharge, ${ }^{(5-8)}$ electron beam excitation, ${ }^{(9)}$ electron beam sustained discharge, ${ }^{(3,4)}$ fission fragment excitation, ${ }^{(10)}$ and microwave discharge. ${ }^{(11)}$

Power deposition from 10 to $10^{6} \mathrm{~W} / \mathrm{cm}^{3}$ atm., pulse durations of $10 \mathrm{~ns}$ to $5 \mathrm{~ms}$, and pressures of $0.5-14$ bar in mixtures with fractions of $10^{-4}$ to $10^{-1}$ rare gas lasing atoms in rare gas buffers have been investigated. So far the most promising results with respect to power and efficiency were obtained for an Ar-Xe mixture using electron beam or electron beam sustained discharges.

Parallel to these performance experiments there has been great interest in the better understanding of the kinetic processes of these systems..$^{(9,12-14)}$ The electro-ionization laser turns out to be a complicated system. The excitation and quenching mechanisms are still subject of extensive studies. Both molecular and electronic collisions play an important role in the production as well as the quenching of the laser levels. In the following we shall give a survey of successful electro-ionization laser systems and discuss the kinetics of the laser process. Especially we pay attention to the performance of an e-beam sustained system with the potential of $10 \%$ intrinsic efficiency.

\section{NEAR INFRARED LASING \\ TRANSITIONS IN RARE GAS MIXTURES}

As pointed out in the previous section efficient laser action on infrared transitions of rare gas requires homogeneous discharges at multi-atmosphere. Since the excitation of the upper laser level is a consequence of the atomic recombination by three-body collisons it is from a scientific point of view attractive to study the laser process within a large pressure regime independent on discharge conditions. This can be achieved with e-beam or e-beam sustained systems. The latter systems are more complicated and may have a lower upper pressure limit than pure e-beam systems. Especially coaxial systems have been developed to yield high pressures up till 14 bar under e-beam discharge conditions. ${ }^{(15)}$ Using a small coaxial electron beam apparatus with an active volume of $13.3 \mathrm{~cm}^{3}$ various rare gas mixture have been investigated. ${ }^{(16)}$ Each mixture contained a low fraction of either $\mathrm{Ar}, \mathrm{Kr}$ and $\mathrm{Xe}$ as the lasing gas diluted in either $\mathrm{Ar}, \mathrm{Ne}$ or $\mathrm{He}$ as a buffer gas. The energy deposition 


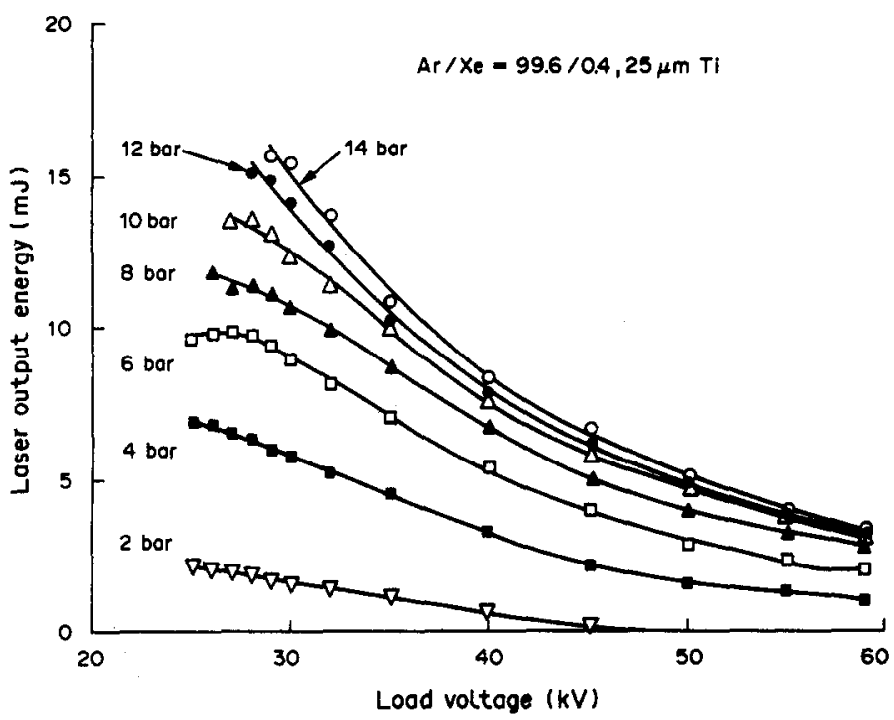

Fig. 3. Output energy from a coaxial e-beam apparatus of $13.3 \mathrm{~cm}^{3}$ active volume filled with $0.4 \%$ of Xe in $\mathrm{Ar}$ as a function of the load voltage per stage of a 10 stage Marx generator is plotted for various pressures.

in the laser mixture by the e-beam as it depends on the gas pressure can be varied from $3 \mathrm{MW} / \mathrm{cm}^{3}$ at 1 bar to $20 \mathrm{MW} / \mathrm{cm}^{3}$ at 10 bar. Studying the $\mathrm{Ar}-\mathrm{Xe}$ mixture with $\mathrm{Ar}$ as the lasing gas the optimum conditions are obtained for $0.3-0.5 \% \mathrm{Xe}$. In Fig. 3 the output for $0.4 \% \mathrm{Xe}$ in Ar is plotted as a function of the load voltage per stage of a 10 stage Marx generator. It is interesting to observe the increasing output with decreasing energy deposition. We shall discuss these results further on. Compared with other rare gas compositions the Ar-Xe mixture gives the highest output power. Replacing the buffer gas Ar by He or Ne results to less output power. Studying the behaviour of $\mathrm{Kr}$ and $\mathrm{Ar}$ as the lasing gas it is found that $\mathrm{He}$ as buffer gas gives the best results. ${ }^{(16)}$ The output increases with pressure and reaches a maximum at about 10 bar. The main wavelengths observed with these systems are shown in Table 1.

\section{QUENCHING OF THE INVERSION DENSITY BY THE DISCHARGE ELECTRONS}

It was observed as shown in Fig. 3 that the optimum output power will be generated at relatively low power deposition. ${ }^{(13.16)}$ Further it was observed that for high deposition in short pulses (about $30 \mathrm{~ns}$ ) there is for most laser transitions a delay between the start of the current pulse and the start of the laser oscillation as a function of the power deposition. This peculiar behaviour of maximum performance for low energy deposition and delay times has been interpreted by electron collision mixing effects. ${ }^{(13,17)}$ A computer model was used to analyse the observations and to predict the electron and heavy particle kinetics. The kinetics shows that electron collisions quench the inversion by coupling the upper $5 \mathrm{~d}$ laser levels to the lower $6 \mathrm{p}$ laser levels. The occupations of all levels in the $6 \mathrm{p}$ and $5 \mathrm{~d}$ manifolds have by the collisional coupling the tendency to thermalize and thus to destroy the inversion. Thus on one hand the discharge electrons recombine with the ions to produce the upper state and on the other hand the electrons quench the inversion. This means that after the e-beam pulse the laser does not begin to oscillate until the electron density falls below a value for which the quenching is sufficiently low and the recombination rate is still sufficient to obtain

Table 1. Wavelengths measured for different gas mixtures

\begin{tabular}{llllll}
\hline & \multicolumn{5}{c}{ Wavelength $[\mu \mathrm{m}]$} \\
\hline $\mathrm{He}-\mathrm{Ar}$ & 1.79 & & & & \\
$\mathrm{He}-\mathrm{Kr}$ & 2.52 & 3.07 & & & \\
$\mathrm{He}-\mathrm{Xe}$ & 2.03 & 2.65 & 3.65 & & \\
$\mathrm{Ar}-\mathrm{Xe}$ & 1.73 & 2.03 & 2.63 & 2.65 & 3.37 \\
\hline
\end{tabular}




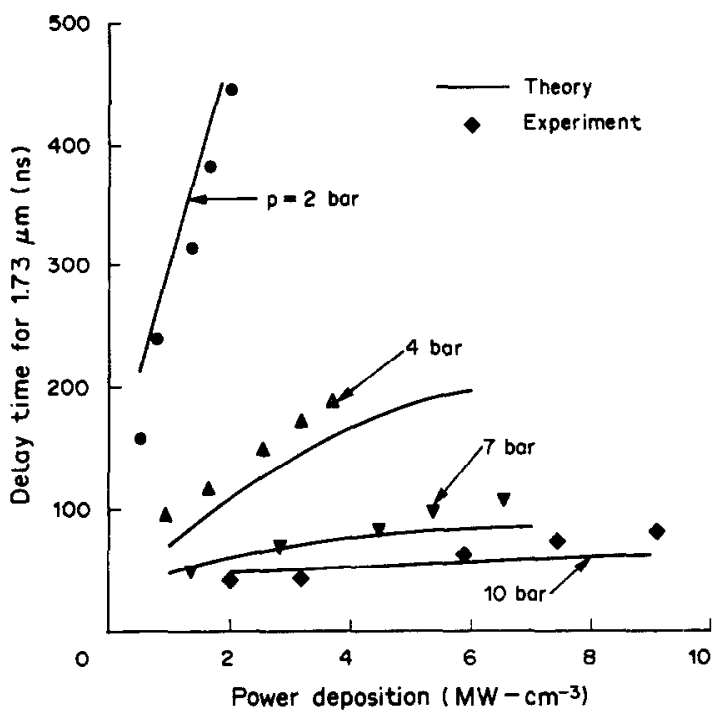

Fig. 4. The delay time for the $1.73 \mu \mathrm{m}$ oscillation after the start of the current pulse in a Ar-Xe mixture of $0.3 \%$ Xe as a function of average power deposition during a $30 \mathrm{~ns}$ excitation pulse. ${ }^{(13)}$

inversion. These delay times for the $1.73 \mu \mathrm{m}$ transition of xenon are plotted in Fig. 4 together with the calculated values. A good agreement with the experiments is obtained. ${ }^{(13)}$

\section{INTRINSIC EFFICIENCY AND CRITICAL POWER DEPOSITION OF THE ELECTRO-IONIZATION LASER}

Experiments revealed that pure e-beam excitation is not the best way for obtaining optimum performance. This is not only because of fractional losses through the transmitting foil but because the e-beam ionizes mainly the atoms in the ground state. Much better performance is obtained with an e-beam sustained discharge in which a low current density of the e-beam is only used to maintain the stability of the discharge and to supply a reservoir of metastable atoms to be used in the kinetic chain of the laser process. These metastables serve as the "ground level" of a four level laser cycle that is driven by the sustainer. A scheme of such a device is shown in Fig. 5. The ionizing electrons reaching the foil of the e-beam are in the range of $100-300 \mathrm{keV}$. The sustainer capacitor connected to the electrodes is charged to twice the quasi steady state discharge voltage of the system. It is observed $^{(4)}$ that the discharge characteristic shows a nearly constant voltage between the electrodes over a wide current range. It can be shown that for a sustainer charging voltage equal to twice this discharge voltage the total sustainer energy can be transferred to the discharge medium without current reversal i.e. current oscillations from the sustainer. In this case the current density depends

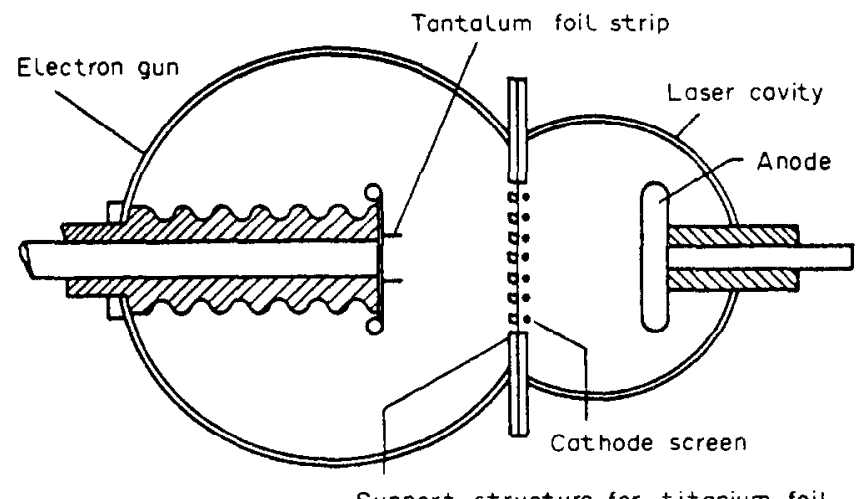

Fig. 5. Cross sectional view of an e-beam sustained discharge. The optical axis of the laser cavity is perpendicular to the figure. 


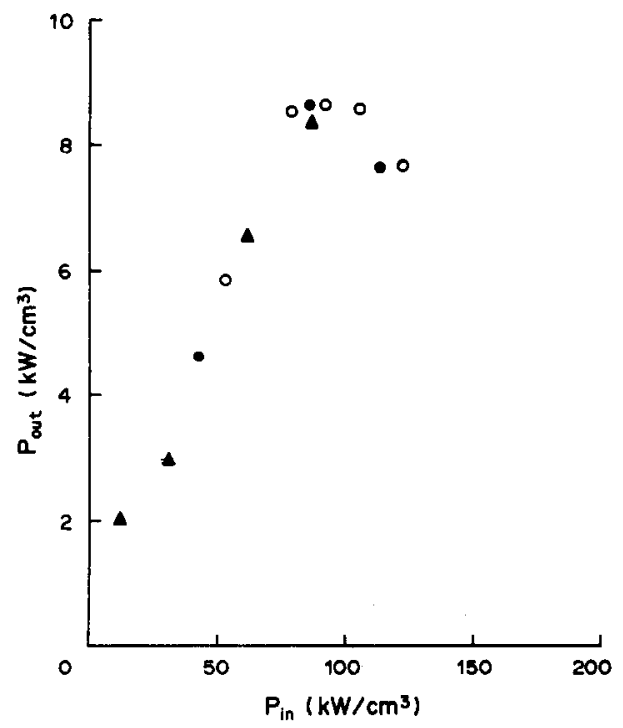

Fig. 6. Output power of a 7 bar e-beam sustained Ar-Xe laser as a function of power deposition. The sustainer circuit contains a capacitor of $5.4 \mu \mathrm{F}$ and serial resistor of 0.06 $(\bigcirc), 0.1(\bigcirc)$ and $0.5 \Omega(\triangle)$.

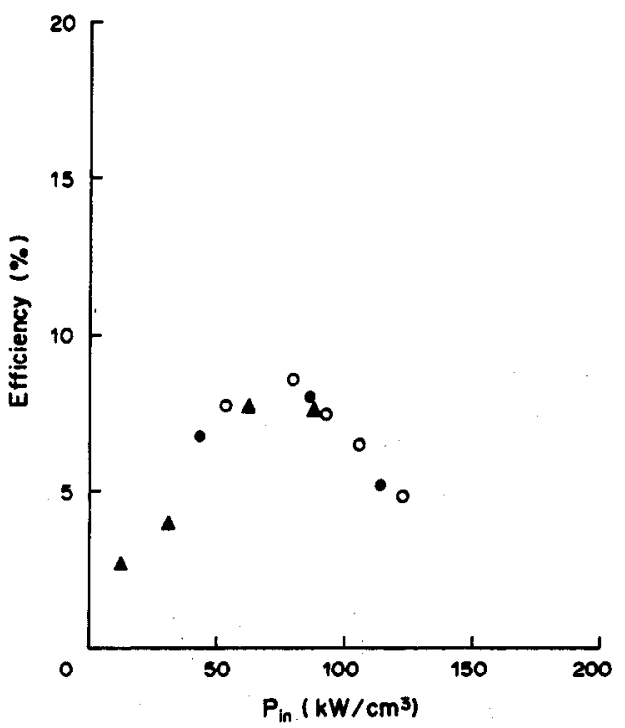

Fig. 7. Intrinsic efficiency of a 7 bar e-beam sustained $\mathrm{Ar}-\mathrm{Xe}$ laser as a function of power deposition. The results are deduced from Fig. 6.

on the LC-value of the circuit. In order to investigate the sustainer parameters such as current density and pulse duration the sustainer circuit contains a variable serial resistor and a variable capacitor so that a range of current densities and pulse durations can be observed. We used an active volume of $330 \mathrm{~cm}^{3}$ and an aperture of $5.9 \mathrm{~cm}^{2}$ at 7 bar with $0.4 \%$ xenon in Ar. The e-beam pulse width was $1.1 \mu \mathrm{s}$ with a current density of $120 \mathrm{~mA} / \mathrm{cm}^{2}$ and energy of $180 \mathrm{keV}$ behind the foil. Three resistor values of $0.06,0.1$ and $0.5 \Omega$ were used with a capacitor of $5.4 \mu \mathrm{F}$. In this way a large current and sustainer pulse duration are obtained. The results show that the output power is independent of the parameter choice of resistor or capacitor but depends only on the discharge current. Further the highest output is obtained when the e-beam and sustainer pulse are simultaneously present. After the termination of the e-beam the sustainer discharge becomes much less effective in the laser process. During the e-beam the output power is more or less constant. The observed output powers with the above described system are plotted in Fig. 6. The corresponding efficiencies are shown in Fig. 7. It was observed that even for an e-beam pulse of $30 \mu$ s the output pulse duration corresponds to the duration of the e-beam. ${ }^{(3)}$

It is seen that the output power saturates with the deposited power i.e. for more or less constant voltage it saturates with current. We suggest ${ }^{(4)}$ that this saturation is also caused by the above mentioned electron collision mixing of the manifolds of the upper and lower laser levels. The lower laser level is depopulated by spontaneous and collision induced decay to the metastable state. At increasing excitation rates the lower laser level density increases and subsequently the upper laser level density must increase with the same amount. The higher the upper state density the higher the relaxation rate of this state by electron collision mixing and the less the available fraction for the stimulated emission process. Thus both the electron density and the density of the upper laser level increase with current so that the relaxation losses increases and the output will saturate with current.

\section{CONCLUSIONS}

The electro-ionization laser is a fascinating near-infrared light source and it has the potential of high power operation. Intrinsic efficiencies of $8 \%$ are demonstrated. For several reasons e.g. wavelength region, no thermal population of lower laser level, reproducible discharge, high efficiency, this system may also be an attractive candidate for high power applications. The complicated technology of e-beam sustained operation may give limitations with respect to repetition rate and pulse length. However, further development on foil constructions, its cooling provisions and minimum required e-beam current density may release these limitations. 


\section{REFERENCES}

1. L. A. Newman and T. A. DeTemple, Appl. Phys. Lett. 27, 678 (1975)

2. S. A. Lawton, J. B. Richards, L. A. Newman, L. Specht and T. A. DeTemple, J. appl. Phys, 50, 3880 (1979).

3. N. G. Basov, V. V. Baranov, A. Y. Chugunov, V. A. Danilychev, A. Y. Dudin, I. V. Kholin, N. N. Ustinoviskii and D. A. Zayarnyi, IEEE J. Quant. Electron QE-21, 1756 (1985).

4. H. Botma, P. J. M. Peters and W. J. Witteman, Appl. Phys. To be published.

5. S. E. Schwarz, T. A. DeTemple and R. Targ, Appl. Phys. Lett. 17, 305 (1970).

6. O. R. Wood, E. G. Burkhardt, M. A. Pollack and T. J. Bridges, Appl. Phys. Lett. 18, 261 (1971).

7. F. S. Collier, P. Labastie, M. Maillet and M. Michon, IEEE J. Quant. Electron QE-19, 1129 (1983).

8. J. E. Tucker, B. L. Wexler, B. J. Feldman and T. McClelland, IEEE Phot. Tech. Lett. 1, 193 (1989).

9. P. J. M. Peters, Qi-Chu Mei and W. J. Witteman, Appl. Phys. Lett. 54, 193 (1989).

10. W. J. Alford and G. N. Hays, J. appl. Phys. 65, 3760 (1989).

11. C. L. Gordon III, B. Feldman and C. P. Christensen, Opt. Lett. 13, 114 (1988).

12. N. G. Rasov, A. Y. Chugunov, V. A. Danilychev, I. V. Kholin and M. N. Ustinovsky, IEEE J. Quant. Electron QE-19, 126 (1983).

13. P. J. M. Peters, Yun Fu Lan, Mieko Ohwa and Mark J. Kushner, IEEE J. Quant. Electron. In press.

14. Y. F. Lan, P. J. M. Peters and W. J. Witteman. To be published.

15. P. J. M. Peters, I. H. T. Fierkens, W. J. Witteman, Appl. Phys. Lett. 51, 883 (1987).

16. P. J. M. Peters, MEI Qi-Chu and W. J. Witteman, Appl. Phys. B47, 187 (1988).

17. M. Ohwa, T. J. Moratz and M. J. Kushner, J. appl. Phys. 66, 5131 (1989). 